\section{Quantification of placental change in mouse models of preeclampsia using magnetic resonance microscopy}

Gabriele Bobek, ${ }^{1,2}$ Tim Stait-Gardner, William S. Price, ${ }^{1,2}$ Angela Makris, ${ }^{1,3,4}$ Annemarie Hennessy ${ }^{1,2,3,4}$

${ }^{1}$ School of Medicine, Western Sydney University, Campbelltown

${ }^{2}$ Nanoscale Organisation and Dynamics, School of Science and Health, Western

Sydney University, Campbelltown

${ }^{3}$ The Heart Research Institute, University of Sydney

${ }^{4}$ Liverpool Hospital, Renal Unit, Liverpool, NSW, Australia

\section{Abstract}

Abnormal development of the placenta is postulated to be central to the aetiology of preeclampsia. This study investigates changes in placental histopathology in mouse models of preeclampsia compared to the morphology using magnetic resonance microscopy (MRM) (11.7 T) of intact ex vivo tissue followed by $3 \mathrm{D}$ analysis of the image data. Here, C57BL/6JArc pregnant mice were subject to either normal pregnancy $(n=3)$, or to one of two experimental models of preeclampsia; TNF- $\alpha$ infusion $(n=3)$ or reduced uterine perfusion pressure (RUPP) ( $\mathrm{n}=3)$. Placental tissue was collected at gestational day ( $\mathrm{gd}$ ) 17, fixed in formalin and incubated with Magnavist ${ }^{\mathrm{TM}}$ contrast agent, and high resolution images (50 $\mu \mathrm{m} \times 50 \mu \mathrm{m} \times 50 \mu \mathrm{m}$ voxels) obtained by magnetic resonance imaging at $11.74 \mathrm{~T}$. Visual segmentation into placental subregions and three dimensional $(3 \mathrm{D})$ reconstruction followed by volume analysis was performed with Amira ${ }^{\mathrm{TM}}$ 3D analysis software. The significance of differences between treatment groups in total and regional volumes was assessed. In a single placenta the volumes measure by standard histology were compared. Three placentas from each animal were imaged, segmented into anatomical regions and 3D reconstructions generated. Total placental volume, labyrinth and decidual volume were not significantly different between groups. The junctional zone volume was found to be significantly larger in the RUPP animals $\left(18.5 \pm 1.5 \mathrm{~mm}^{3}\right)$ compared to TNF- $\alpha$ infused animals $(15.8 \pm 1.5)$ or control animals (15.0 $\pm 0.7, \quad \mathrm{P}<0.01)$. However, the decidual/junctional zone volume was smaller in the TNF- $\alpha$ compared to control ani- mals $(\mathrm{P}<0.05)$. Placental structural change in experimental models of preeclampsia is able to be visualized and quantified using MRM and 3D analysis. These techniques could prove to be a powerful tool in examining changes in placental morphology.

\section{Introduction}

Preeclampsia, a syndrome characterised by hypertension and proteinuria is the most significant and common complication of pregnancy, affecting 3-5\% of all pregnancies. ${ }^{1}$ Abnormal development of the placenta, which serves as the interface between the maternal and foetal environments, is postulated to be central to the aetiology of preeclampsia. $^{2}$ The placentas of women with preeclampsia have evidence of reduced perfusion due to failure of the spiral artery remodelling. Placental infarcts and atherosis may be evident on histopathological analysis. ${ }^{3}$ Cytokines have also been shown to be inhibitors of the placental vascular remodelling, where pro-inflammatory molecules imbalance (e.g., IL-6 or LPS) inhibits trophoblast invasion. ${ }^{4,5}$ Preeclampsia occurs only very rarely in animals pregnancies and as such animal models have become necessary to further investigate this disease. Many rodent models have been generated but not all manifest all of the clinical signs of human preeclampsia. Of all the inflammatory models, TNF $\alpha$ infusion has been the best studied and replicates many of the clinical and biochemical findings of human preeclampsia eg proteinuria, hypertension, reduced renal function, increased endothelin-1. The reduced-uterine perfusion pressure (RUPP) model also develops the appropriate clinical and biochemical signs and mimics the placental hypoperfusion seen in de novo disease. The structural anatomy of the normal mouse placenta has been documented using plastic vascular casts and serial histological sections $^{6}$ and changes during normal mouse placental development have been assessed using conventional stereological techniques. ${ }^{7}$ Such techniques use unbiased sampling and estimation tools to estimate three dimensional (3D) parameters of tissues such as volume from 2D histological sections. ${ }^{8}$ Analysis of histological sections is subject to artefacts introduced by the tissue processing and sectioning. The advent of magnetic resonance (MR) scanners with much stronger magnets $(>7 \mathrm{~T})$ than clinical magnetic resonance imaging (MRI) scanning capacity (usually $1.5-3.0 \mathrm{~T}$ ) has led to the development of imaging with a spatial resolution of $<100 \mu \mathrm{m}$, termed MR microscopy (MRM). ${ }^{9}$ Using MRM of isolated intact organs has the benefit of cir-
Correspondence: Prof. Angela Makris, School of Medicine, Western Sydney University Building 30, Goldsmith Avenue, Campbelltown NSW 2757, Australia.

Tel. +61.2.46203903 - Fax: +61.2.46203618. E-mail: angela.makris@sswahs.nsw.gov.au

Key words: Placental volume; preeclampsia; MRI; Magnetic Resonance Microscopy; 3-D reconstruction.

Acknowledgements: The authors would like to gratefully acknowledge the statistical assistance of Associate Professor J. Lind, Western Sydney University, Australia.

Funding This work was supported by grant APP 1025258 from the National Health and Medical Research Council, Australia. It was also supported by resources supplied by the Australian National Imaging Facility (NIF).

Received for publication: 6 November 2017. Accepted for publication: 26 February 2018.

This work is licensed under a Creative Commons Attribution-NonCommercial 4.0 International License (CC BY-NC 4.0).

(C) Copyright G. Bobek et al., 2018

Licensee PAGEPress, Italy

European Journal of Histochemistry 2018; 62:2868 doi:10.4081/ejh.2018.2868

cumventing such tissue distortions while providing non-destructive high spatial resolution comparable to histology. ${ }^{10,11}$ The typical mouse placenta has a diameter of 9-10 $\mathrm{mm}$ and a depth of 4-5 $\mathrm{mm}$, with morphological regions of the placenta such as the labyrinth or junctional zone having typical depths of $1.5 \mathrm{~mm}$ and $400 \mu \mathrm{m}$, respectively. As such, the improved MRM technology has the ability to resolve these structures.

This study aims to compare and measure changes in structural features of the placental in two established models of preeclampsia; the reduced uterine perfusion pressure (RUPP) model and the TNF- $\alpha$ infusion model $^{12,13}$ using MRM images of the intact complete ex vivo placenta followed by reconstruction of a 3D model of the placenta from the data set.

\section{Materials and Methods}

\section{Ethics statement}

All procedures were approved by the Western Sydney University Animal Care and Ethics Committee and follow the "Guidelines to Promote the Wellbeing of Animals used for Scientific Purposes" as laid out by the National Health and Medical Research Council of Australia, (2008). 


\section{Animals}

The C57BL/6JArc mice were obtained from the Animal Resource Centre (Canning Vale, WA, Australia) and were housed in a temperature-controlled room in individually ventilated cages (up to 5 per cage), maintained in a 12:12-h light-dark cycle with ad libitum access to water and standard rodent chow. Animals were time-mated and on day 13.5 of gestation were randomly assigned to either a RUPP procedure $(n=3)$, TNF- $\alpha$ infusion $(n=3)$ or control normal pregnancy $(n=3)$ as previously described, ${ }^{13}$ and housed individually until tissue collection on gestational day 17.5. Upon euthanasia of the animal by cervical dislocation, the uterus was dissected from the mouse and intact embryo placental units (EPU) within the uterine horn were collected for MRM.

\section{Experimental mouse models of preeclampsia}

Both the RUPP procedure and TNF- $\alpha$ infusion have been utilized as experimental models of preeclampsia in baboons ${ }^{14,15}$ and rats. ${ }^{16,17}$ For this murine study the RUPP procedure and TNF $\alpha$ infusion was performed as previously described. ${ }^{13}$ Briefly, for RUPP a silk suture was tied around the right uterine artery proximal to the ovarian artery on day 13.5 of gestation. For TNF- $\alpha$ infusion, a mini-osmotic pump (Model 1007D, Alzet, Cupertino, CA) primed with mouse recombinant TNF- $\alpha$ (Bio-Scientific Pty Ltd, Kirrawee, Australia) was subcutaneously implanted below the right scapulae on day 13.5 of gestation. The pump was designed to deliver a constant release of $\mathrm{TNF}-\alpha(500 \mathrm{ng} / \mathrm{kg} /$ day) from implantation until euthanasia at gestational day 17.5. Surgical anaesthesia for both procedures was induced with $4 \%$ isofluorane and maintained at $2 \%$ during surgery. Analgaesia was administered pre-operatively via subcutaneous injection (buprenorphine $0.1 \mathrm{mg} / \mathrm{kg}$ ).

\section{Preparation of fixed placenta for magnetic resonance microscopy}

To enable the long scan times required for MRM and to preserve the native shape of the placenta, the isolated placentas were fixed while still part of the intact EPU. The EPU within the uterine horn were rinsed in ice-cold phosphate buffered solution (PBS) then fixed in $10 \%$ formalin (Sigma-Aldrich, Sydney, Australia) at $4{ }^{\circ} \mathrm{C}$ for 14 days. Following fixation, the EPU were rinsed briefly with PBS pH 7.4 and dissected to isolate the placenta and associated mesometrium. The isolated placentas were incubated with $2 \mathrm{mM}$ dimeglumine gadopentetate (Magnevist $^{\mathrm{TM}}$ ) (Bayer Healthcare, Pymble, NSW, Australia) for a further 10 days. The placentas were embedded in $1 \%$ agarose gel containing $2 \mathrm{mM}$ Magnevist in a $10 \mathrm{~mm}$ thin wall nuclear magnetic resonance (NMR) sample tube (Wilmad Lab Glass, NJ, USA) ready for imaging.

\section{Magnetic resonance microscopy of fixed placenta}

The ${ }^{1} \mathrm{H}$ MRI images were taken of the fixed placentas using a Bruker Avance 11.74 Tesla wide-bore spectrometer with micro-imaging probe (micro 2.5) capable of generating gradients of $1.5 \mathrm{~T} / \mathrm{m}$. A Gradient Echo Fast Image (GEFI) sequence was used to obtain a series of contiguous images across the complete fixed placenta ( 3 placentas from each of 3 dams per group, totalling 9 placentas per treatment group) using the following parameters; echo time 8 $\mathrm{ms}$, repetition time $40 \mathrm{~ms}$, excitation pulse angle 58.4 degrees, 16 averages with 3D 50 $\mu \mathrm{m}$ isotropic voxels, and typically a field of view (FOV) of $11 \mathrm{~mm}$ x $11 \mathrm{~mm}$ x $20 \mathrm{~mm}$ although the longitudinal dimension was varied depending on the number of placentas to be scanned and a matrix size (MTX) of typically $220 \times 220 \times 400$ (the longitudinal MTX was chosen so as to ensure $50 \mu \mathrm{m}$ isotropic voxels for every scan). Scans typically took $16 \mathrm{~h}$.

\section{Creation and analyses of placental maps}

Analyses of the placental images were performed with Amira ${ }^{\mathrm{TM}}$ 3D Analysis software (FEI Visualization Sciences Group, Mérignac Cedex, France). The MRI image sets from each placenta were imported into the software and segmented into regions; amnion, labyrinth, junctional zone, decidua, and mesometrium, on the basis of areas of image contrast and the known anatomy of mice placenta. Images were filtered and converted to a gradient image to enable automatic segmentation based on Canny edge detection, ${ }^{18}$ which uses a multi-stage algorithm to detect edges in images based on intensity gradients. Marker voxels for all regions were assigned and marker based watershed segmentation was performed. Each slice was checked for assignment of voxels into regions and manual adjustment of voxels was performed if required. Volume rendering (Volren) of the various segments of the placenta (as described above) were created and volume analysis performed.

\section{Stereological analysis by histology}

In order to validate the volume estimates obtained from the MRM images, following MRM placentas were subject to volume estimation by conventional stereology of histological sections. All 4 placentas from a randomly selected single animal were removed from the NMR tube, processed into paraffin blocks and subject to conventional stereological analysis as follows. Serial sections $(10 \mu \mathrm{m})$ across each complete placenta were cut and every $40^{\text {th }}$ section was collected and stained with Haematoxylin and Eosin (H\&E) (Grale HDS, Wetherill Park, Australia). An Axio Imager II microscope (Carl Zeiss Microscopy GmbH, Jena, Germany) was used to acquire images at total magnification of $50 \times$. Images taken at total magnification of $630 \times$ were used to measure red blood cell diameter in order to correct for shrinkage during processing. Stereological analysis of H\&E stained sections were performed with Steroinvestigator software (MBF Biosciences, Williston, VT, USA) using the Cavilleri probe to estimate volumes of placental regions. A grid size of $250 \mu \mathrm{m}$, section thickness of $10 \mu \mathrm{m}$ and section evaluation interval of 40 was used and corrections for shrinkage during processing were made based on \% red blood cell shrinkage.

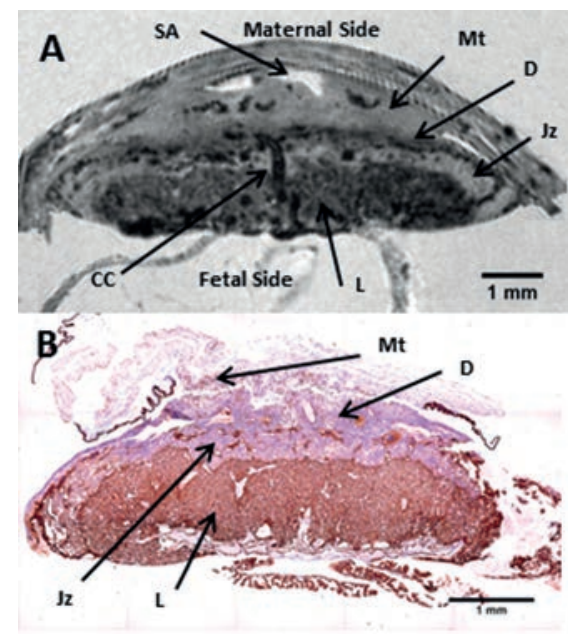

Figure 1. A) Representative high resolution $(50 \times 50 \times 50 \mu \mathrm{m}$ voxels $)$ GEFI image of a formalin fixed and Magnevist contrast agent infused gestational day 17 control placenta. B) Representative histological section immunostained with cytokeratin showing trophoblasts (brown) (100x magnification) (reproduced with permission from Bobek et al., PLoS One 2013;8:e59971). Regions identified in MRI image correspond to those in histological section; Labyrinth (L), Junctional zone (Jz) comprising spongiotrophoblast and glycogen trophoblast cells, the decidua (D) and the mesometrial triangle (mesometrium, Mt). Other features observed are central canal (CC) and spiral arteries (SA). Note that shrinkage of $\sim 1.5$ for the tissue processed for histology and the distortions of tissue especially the mesometrium. 


\section{Statistics}

Statistical analysis was carried out using SPSS software (ver. 20) (SPSS, Inc., Chicago, IL, USA). Generalized Estimating Equation Modelling clustering placenta within animals and animals within treatment groups was used to evaluate the differences in volume measurements and the labyrinth/junctional and junctional zone/ decidua ratios. Data are expressed as means \pm SEM with the level of significance being $\mathrm{P}<0.05$. Data distribution that was nonparametric was normalised by logarithmic transformation prior to statistical analysis. For the study comparing volume measurements made by MRI or conventional stereology, measured volumes in paired placenta were compared using a paired samples $t$-test $(\mathrm{P}<0.05)$. Litter sizes and pup weights were compared using Student's $t$-test $(\mathrm{P}<0.05)$.

\section{Results}

All animals included in the study had healthy pregnancies and displayed no visual or behavioural signs of illness. Litter numbers and pup weights were not significantly different between control, TNF- $\alpha$ or RUPP animals (Table 1).

\section{Magnetic resonance microscopy of mice placenta identifies placenta regions}

High resolution GEFI of isolated fixed placentas were obtained (Figure 1a) where many of the structural features of the mice placenta could be identified including the labyrinth, junctional zone, decidua and metrial triangle. The central canal and spiral arteries are clearly distinguished, with reso- lution comparable with low magnification optical bright-field microscopy (Figure 1b). Notable are the absence of tissue distortions and shrinkage that are artifacts of processing and sectioning for histology.

\section{Segmentation of placental images}

Each slice of the contiguous series of images for each placenta was segmented into placental regions based on image contrast and the known anatomy of the placenta (Figure 2). A combination of semi-automated methods based on segmented data. Figure 3 shows representative images of placenta from animals from each treatment group with coloured overlays depicting the segmented data.

\section{Reconstruction of 3D models of the placenta}

$3 \mathrm{D}$ reconstruction of the placentas was performed using the segmented label data. Each of the labelled regions are able to be visualised independently and can be combined to form a complete 3D model enabling quantitative assessment of structural features such as the labyrinth, junctional zone, decidua and mesometrium. Figure 4 shows the Volren (volume render) of a representative reconstructed placenta.

\section{Volumetric measurements of placental regions}

Volumetric analysis of the segmented placental regions showed that the junctional zone in RUPP animals was significantly larger compared to control animals (Table $2, \mathrm{P}<0.01)$. The measured ratio of labyrinth volume/junctional zone volume was significantly smaller in RUPP mice than control animals $(\mathrm{P}<0.01)$ (Figure 5a). The ratio of the decidua volume/junctional zone volume was significantly smaller in TNF- $\alpha$ animals than control animals $(\mathrm{P}<0.05)$ with a trend for a smaller decidua volume/junctional zone volume ratio in RUPP animals (Figure $5 b)$. While measurements of amnion and mesometrial volumes were performed and are presented here for completion, it is noted that the volume estimations represent the amount of this tissue that remained attached to the placenta during dissection, and as such are highly variable.

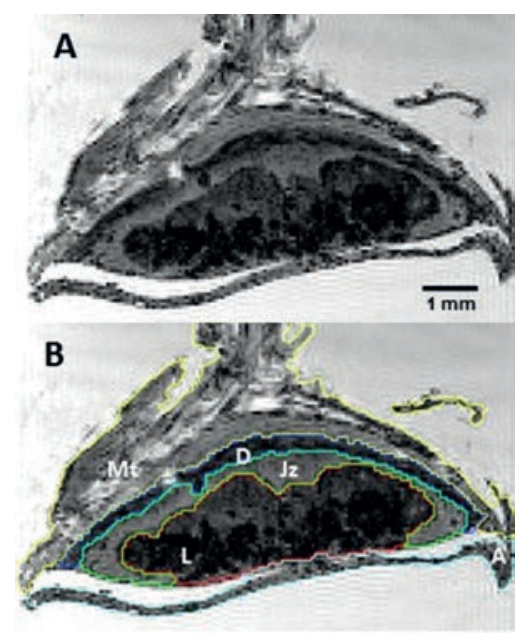

Figure 2. Representative GEFI image (A) of a single 'virtual slice' of a placenta from a TNF- $\alpha$ infused animal and (B) overlay showing segmentation into placental regions as indicated. $\mathrm{Mt}$, mesometrium; $\mathrm{D}$, decidua; Jz, junctional zone; L, labyrinth; A, amnion. Segmentation was performed using the visualisation software (Amira ${ }^{\mathrm{TM}}$ ).

Table 1. Litter sizes and Embryo Placental Unit (EPU) weights.

\begin{tabular}{lccc} 
Treatment group & $\begin{array}{c}\text { Mean pup number } \\
\text { (range) }\end{array}$ & $\begin{array}{c}\text { Mean total EPU } \\
\text { weight }(\mathrm{g}) \text { (range) }\end{array}$ & $\begin{array}{c}\text { Mean weight EPU/pup } \\
(\mathrm{mg})(\mathrm{range})\end{array}$ \\
Control $(\mathrm{n}=3)$ & $7(6-8)$ & $9.2(8.5-9.7)$ & $1.39(1.17-1.52)$ \\
TNF- $\alpha(\mathrm{n}=3)$ & $6.8(5-9)$ & $9.4(6.0-10.9)$ & $1.27(1.21-1.31)$ \\
\hline RUPP $(\mathrm{n}=3)$ & $7.7(6-9)$ & $9.9(7.8-11.7)$ & $1.29(1.27-1.31)$ \\
\hline
\end{tabular}

Comparison between treatment groups was made using Students t-test, P-value not significant.

Table 2. Volume of placental regions.

\begin{tabular}{|c|c|c|c|c|c|c|}
\hline \multirow[t]{2}{*}{ Treatment group } & \multicolumn{3}{|c|}{ Volume \pm SEM $\left(\mathrm{mm}^{3}\right)$} & \multirow[b]{2}{*}{ Decidua } & \multirow[b]{2}{*}{ Metrial } & \multirow[b]{2}{*}{ Total $(L+J+D)$} \\
\hline & Amnion & Labyrinth & Junctional zone & & & \\
\hline Control ( $\mathrm{n}=9)$ & $9.7 \pm 1.27$ & $38.9 \pm 0.9$ & $15.0 \pm 0.7$ & $20.1 \pm 0.9$ & $22.0 \pm 3.0$ & $96.2 \pm 5.9$ \\
\hline TNF- $\alpha(n=9)$ & $15.8 \pm 0.9$ & $38.1 \pm 1.4$ & $15.8 \pm 1.5$ & $19.2 \pm 1.2$ & $23.2 \pm 2.1$ & $88.7 \pm 11.9$ \\
\hline RUPP (n=9) & $12.99 \pm 1.0$ & $38.0 \pm 0.9$ & $18.5 \pm 1.0 *$ & $21.2 \pm 1.6$ & $19.9 \pm 1$ & $94.6 \pm 9.0$ \\
\hline
\end{tabular}

Generalized Estimating Equation Modelling clustering placenta within animals $(\mathrm{n}=3)$ and animals within treatment groups ( $\mathrm{n}=3$ ) was used to evaluate the statistical differences in volume measurements; ${ }^{*} \mathrm{P}<0.01$. 
Validation of MRM approach compared to conventional stereology

Figure 6 shows a comparison of volume measurements made by MRM and histology. After accounting for shrinkage correction of the histological sections there was no statistical difference between paired measurements made by MRM or conventional stereology except in the mesometrium where tissue distortions upon histological sectioning of this membranous tissue are apparent.

\section{Discussion}

In this study MRM was utilised to create high resolution placental images which identified many of the structural features of the mouse placenta. Imaging by MRM was clearly able to resolve features of the placenta such as the labyrinth, junctional zone, decidua, amnion and mesometrium, as well as vascular features such as the central canal and spiral arteries with dimensions as small as $80-100 \mu \mathrm{m}$ diameter. We demonstrate that MRM data, combined with 3D visualisation software, can be successfully used to create $3 \mathrm{D}$ reconstructions of the mouse placenta with such detail that quanti- tative analysis of regions of the placenta can be performed.

Our results show that the volume of the junctional zone is larger in RUPP placentas, which suffer from uteroplacental malperfusion, while the labyrinth remains unchanged and thus RUPP animals have a smaller labyrinth volume/junctional zone volume ratio. The labyrinth is the functional area of the placenta where nutrient/gas exchange between the foetus and the mother takes place. The junctional zone, which consists of spongiotrophoblast, glycogen trophoblast cells and an outer layer of trophoblast giant cells (TGC's), has a supportive function, acting as both an endocrine compartment and potential energy source. ${ }^{19}$ It is the middle connecting layer of the placenta between the labyrinth and the decidua and gives rise to TGC's and glycogen cells that invade and anchor the placenta to the decidua. ${ }^{20,21}$ Previous studies have shown that the volume of the junctional zone is biphasic across gestation, expanding until gd 16.5 and then reducing by gd $18.5 .^{7}$ Our results suggest that this latter decrease in junctional zone volume may not occur with the malperfused placenta, which may have implications for the cross-talk between the functional and supportive layers of the placenta under stress.
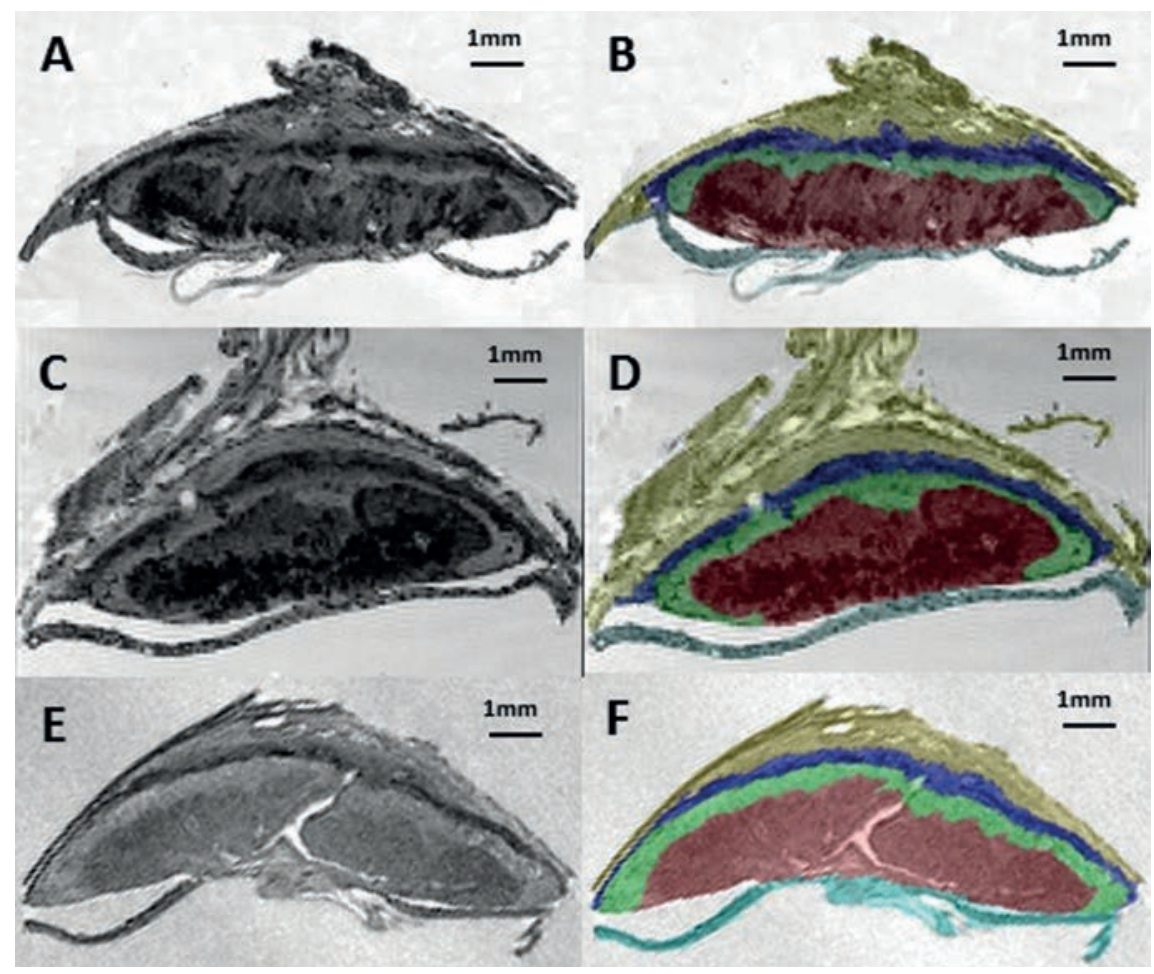

Figure 3. Representative GEFI image of a normal (A,B), TNF- $\alpha$ infused (C,D) and RUPP $(\mathrm{E}, \mathrm{F})$ mouse placenta showing overlay (B,D,F of the segmented labelled regions; mesometrium (yellow), decidua (blue), junctional zone (green), labyrinth (red), amnion (cyan).

The ability to appreciate the changes in tissue anatomy (whilst the tissue is still vital) using MRM compared to histopathology is clinically very helpful. It potentially allows clinicians to directly observe tissue effects of changes in therapy or function. A limitation of our study is that we compared MRI to fixed placenta tissue. Ideally the comparison would have been undertaken with fresh tissue, but this would be very technically almost impossible. However, correlations of functional physiology and fixed placental tissue have been well studied. ${ }^{22,23}$

Validation of volume estimations from MRM showed that estimations were similar to those obtained from histological sectioning and conventional stereological estimations. Images were obtained of comparable resolution with low magnification $(100 \times)$ light microscopy. While cellular detail is not obtained, contiguous images across the complete placenta are acquired and the images are free of the tissue distortions and shrinkage that plague histological sections. This is most apparent for membranous tissues such as the amnion and mesometrium. This study confirms that MRM has the ability of providing high resolution images of intact organs and has the advantage over low resolution light microscopy of allowing ultrastructure delineation without artefactual tissue distortions. It was reassuring that the pathophysiologically different models of preeclampsia (RUPP and TNF- $\alpha$ ) demonstrated different MRI and histopathological changes. The RUPP model, exemplified by reduced perfusion demonstrated changes in the junctional region whereas the inflammatory model (TNF $\alpha$ ) demonstrated most significant changes in the decidua.

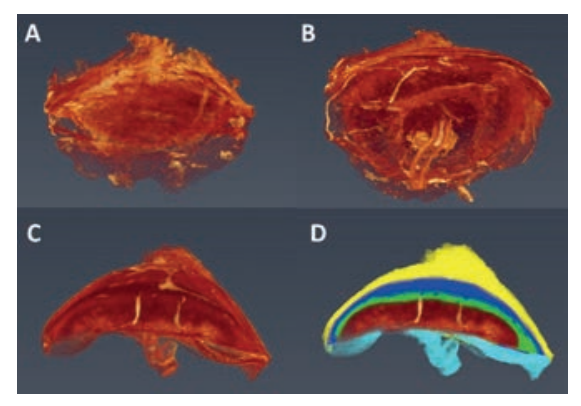

Figure 4. Representative 3D reconstruction of a placenta using segmented label data. Volren of the placenta, showing A) maternal surface view; B) foetal surface view; C) clipped sagittal view; D) clipped sagittal view with solid colours showing mesometrium (yellow), decidua (blue), junctional zone (green), amnion (blue) and the labyrinth remaining in the red density rendering. $3 \mathrm{D}$ volume rendering was performed using the visualisation software $\left(\right.$ Amira ${ }^{\mathrm{TM}}$ ). 
A
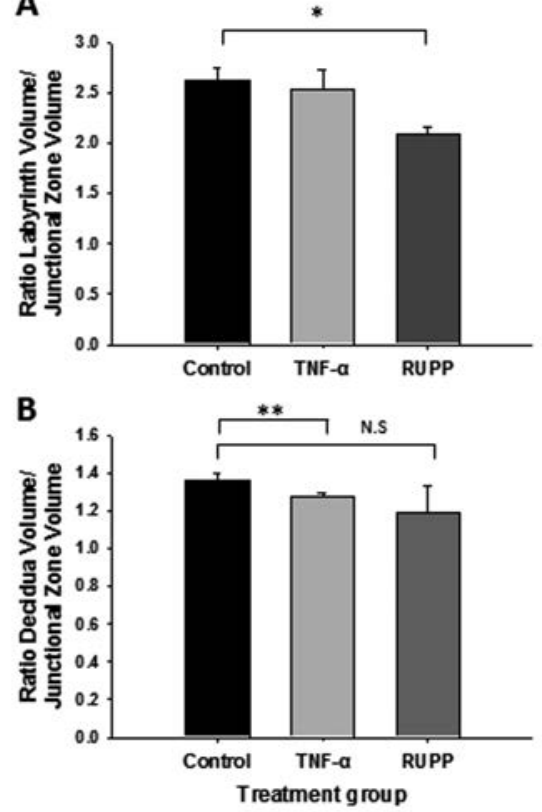

Figure 5. Comparison of ratios of placental region volumes in animals from different treatment groups. A) Labyrinth volume/ Junctional zone volume B) Decidua volume/Junctional zone volume. Generalized Estimating Equation Modelling clustering placenta within animals $(n=3)$ and animals within treatment groups $(n=3)$ was used to evaluate the statistical differences in ratios. A logarithmic transformation was carried out on ratios prior to statistical analysis. ${ }^{*} \mathbf{P}<\mathbf{0 . 0 1}$.

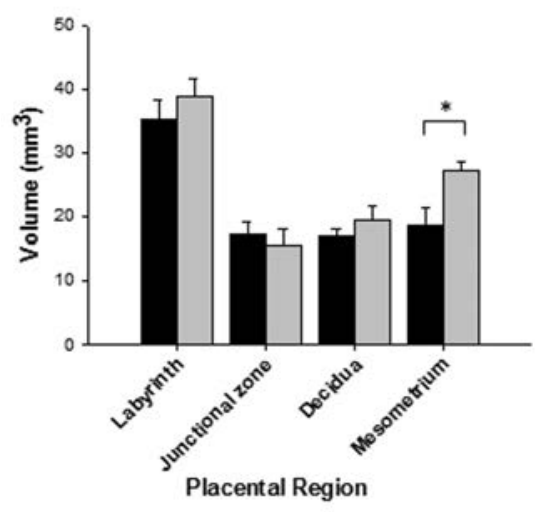

Figure 6. Validation of MRI approach compared to conventional stereology. Four placentas from the same animal were imaged using MRI and then processed into paraffin blocks and sectioned. Volumes estimated by conventional stereology are shown in solid black and volumes measured from the MRI images are shown with diagonal hatched lines. Measured volumes in paired placenta were compared using a paired samples $t$-test $\left({ }^{*} \mathbf{P}<0.05\right)$.
In conclusion, this innovative technique has the potential to visualize and quantify placental structural change in experimental models of preeclampsia. This could prove to be a powerful tool in examining placental morphology and better visualising the dynamic in utero changes in a difficult to sample organ during preeclampsia.

\section{References}

1. Kanasaki K, Kalluri R. The biology of preeclampsia. Kidney Int 2009;76:8317.

2. McMaster MT, Zhou Y, Fisher SJ. Abnormal placentation and the syndrome of preeclampsia. Semin Nephrol 2004;24:540-7.

3. Roberts JM, Gammill HS. Preeclampsia: recent insights. Hypertension 2005;46:1243-9.

4. Champion H, Innes BA, Robson SC, Lash GE, Bulmer JN. Effects of interleukin-6 on extravillous trophoblast invasion in early human pregnancy. Mol Hum Reprod 2012;18:391-400.

5. Anton L, Brown AG, Parry S, Elovitz MA. Lipopolysaccharide induces cytokine production and decreases extravillous trophoblast invasion through a mitogen-activated protein kinase-mediated pathway: possible mechanisms of first trimester placental dysfunction. Hum Reprod 2012;27:6172 .

6. Adamson SL, Lu Y, Whiteley KJ, Holmyard D, Hemberger M, Pfarrer C, et al. Interactions between trophoblast cells and the maternal and fetal circulation in the mouse placenta. Dev Biol 2002;250:358-73.

7. Coan PM, Ferguson-Smith AC, Burton GJ. Developmental dynamics of the definitive mouse placenta assessed by stereology. Biol Reprod 2004;70:180613.

8. Mouton PR. Principles and practices of UNbiased stereology: An introduction for bioscientists. Baltimore: The John Hopkins University Press; 2002.

9. Badea A, Johnson GA. Magnetic resonance microscopy. Stud Health Technol Inform 2013;185:153-84.

10. Driehuys B, Nouls J, Badea A, Bucholz E, Ghaghada K, Petiet A, et al. Small animal imaging with magnetic resonance microscopy. ILAR J 2008;49:3553.

11. Busato A, Fumene Feruglio P, Parnigotto PP, Marzola P, Sbarbati A. In vivo imaging techniques: a new era for histochemical analysis. Eur J
Histochem 2016;60:2725.

12. Bobek G, Surmon L, Mirabito KM, Makris A, Hennessy A. Placental regulation of inflammation and hypoxia after TNF-alpha infusion in mice. Am J Reprod Immunol 2015;74:407-18.

13. Bobek G, Stait-Gardner T, Surmon L, Makris A, Lind JM, Price WS, et al. Magnetic resonance imaging detects placental hypoxia and acidosis in mouse models of perturbed pregnancies. PLoS One 2013;8:e59971.

14. Sunderland NS, Thomson SE, Heffernan SJ, Lim S, Thompson J, Ogle $\mathrm{R}$, et al. Tumor necrosis factor alpha induces a model of preeclampsia in pregnant baboons (Papio hamadryas). Cytokine 2011;56:192-9.

15. Makris A, Thornton C, Thompson J, Thomson S, Martin R, Ogle R, et al. Uteroplacental ischemia results in proteinuric hypertension and elevated sFLT-1. Kidney Int 2007;71:977-84.

16. Alexander BT, Cockrell KL, Massey MB, Bennett WA, Granger JP. Tumor necrosis factor-alpha-induced hypertension in pregnant rats results in decreased renal neuronal nitric oxide synthase expression. Am J Hypertens 2002;15(2 Pt 1):170-5.

17. Alexander BT, Kassab SE, Miller MT, Abram SR, Reckelhoff JF, Bennett WA, et al. Reduced uterine perfusion pressure during pregnancy in the rat is associated with increases in arterial pressure and changes in renal nitric oxide. Hypertension 2001;37:1191-5.

18. Canny J. A computational approach to edge detection. IEEE Trans Pattern Anal Mach Intell 1986;8:679-98.

19. Coan PM, Conroy N, Burton GJ, Ferguson-Smith AC. Origin and characteristics of glycogen cells in the developing murine placenta. Dev Dyn 2006;235:3280-94.

20. Simmons DG, Cross JC. Determinants of trophoblast lineage and cell subtype specification in the mouse placenta. Dev Biol 2005;284:12-24.

21. Soncin F, Natale D, Parast MM. Signaling pathways in mouse and human trophoblast differentiation: a comparative review. Cell Mol Life Sci 2015;72:1291-302.

22. Kingdom J, Huppertz B, Seaward G, Kaufmann P. Development of the placental villous tree and its consequences for fetal growth. Eur J Obstet Gynecol Reprod Biol 2000;92:35-43.

23. Ibrahim NA, Khaled DM. Histological and immunohistochemical study on human placental tissue in normal pregnancy and preeclampsia. Cell Biol 2014;2:72-80. 